\title{
Controllable Chemical Vapour Deposition of Large Area Uniform Nanocrystalline Graphene Directly on Silicon Dioxide
}

Jie Sun ${ }^{\mathrm{a}, *}$, Niclas Lindvall ${ }^{\mathrm{a}}$, Matthew T. Cole ${ }^{\mathrm{b}}$, Teng Wang ${ }^{\mathrm{a}}$, Tim J. Booth $^{\mathrm{c}}$, Peter Bøggild ${ }^{\mathrm{c}}$, Kenneth B. K. Teo ${ }^{\mathrm{d}}$, Johan Liu ${ }^{\mathrm{a}}$, August Yurgens ${ }^{\mathrm{a}}$

${ }^{a}$ Department of Microtechnology and Nanoscience (MC2), Chalmers University of Technology, SE-41296 Gothenburg, Sweden

${ }^{b}$ Electrical Engineering Division, Engineering Department, University of Cambridge, 9 JJ Thomson Avenue, CB3 OFA, Cambridge, United Kingdom

${ }^{c}$ Department of Micro- and Nanotechnology, Technical University of Denmark, 2800 Kgs. Lyngby, Denmark

${ }^{d}$ AIXTRON Nanoinstruments Ltd., Swavesey, Cambridge, CB24 4FQ, United Kingdom

\section{Abstract}

Metal-catalyst-free chemical vapour deposition (CVD) of large area uniform nanocrystalline graphene on oxidised silicon substrates is demonstrated. The material grows slowly, allowing for thickness control down to monolayer graphene. The as-grown thin films are continuous with no observable pinholes, and are smooth and uniform across whole wafers, as inspected by optical-, scanning electron-, and atomic force microscopy. The $s p^{2}$ hybridised carbon structure is confirmed by Raman spectroscopy. Room temperature electrical measurements show ohmic behaviour (sheet resistance similar to exfoliated graphene) and up to $13 \%$ of electric-field effect. The Hall mobility is $\sim 40 \mathrm{~cm}^{2} / \mathrm{Vs}$, which is an order of magnitude higher than previously reported values for nanocrystalline graphene. Transmission electron

\footnotetext{
*Corresponding author. Tel: +46 317723117 .

Email address: jiesu@chalmers.se (Jie Sun)
} 
microscopy, Raman spectroscopy and transport measurements indicate a graphene crystalline domain size $\sim 10 \mathrm{~nm}$. The absence of transfer to another substrate allows avoiding of wrinkles, holes and etching residues which are usually detrimental to device performance. This work provides a broader perspective of graphene CVD and shows a viable route towards applications involving transparent electrodes. 


\section{Introduction}

Graphene, a single-atomic plane of $s p^{2}$ hybridised carbon atoms, is a remarkable material with extraordinary electrical and optical properties by virtue of its unique band structure. The experimentally measured conductance indicates high and approximately equal mobilities for holes and electrons. Graphene is transparent; it absorbs $\pi \alpha \approx 2.3 \%$ of white light, where $\alpha$ is the fine-structure constant [1]. It is expected that graphene will play a crucial role in future nanoelectronics [2] and optoelectronics [3]. Tradi-

tionally, graphene is produced by mechanical exfoliation of graphite [4], a process intrinsically limited to the formation of small flakes (typically a few $\mu \mathrm{m}$ in size) unsuitable for most industrial applications. To date, techniques which are capable of producing large area graphene include epitaxial growth and chemical vapour deposition (CVD). The epitaxial technique where $\mathrm{SiC}$ substrates are heated to high temperatures to sublimate the Si, leaving the $\mathrm{C}$ to form one or more graphene layers [5], is costly due to the quality- and size requirements on the substrates. On the other hand, CVD technique is cost-efficient and scalable. It is compatible with existing semiconductor technologies and is far more realistic for use in industrial processes [6]. In the CVD of graphene, metals such as $\mathrm{Cu}$ [7] or $\mathrm{Ni}[8]$ are commonly used as catalysts. However, since they are electrically conducting, transfer of the synthesised graphene onto insulators is required for most applications. Wrinkles, holes and metal etching residues are inevitable during such transfers and often result in decreased device performance or even failure. For practical applications, a reliable large scale deposition of graphene directly on insulators is highly desirable. Recent advances in a metal-free growth of graphene 
include: CVD (or molecular beam epitaxy (MBE)) on sapphire [9, 10], ZnS [11], $\mathrm{BN}$ [12], GaN [13], $\mathrm{Si}_{3} \mathrm{~N}_{4}$ [14], $\mathrm{MgO}$ [15], and $\mathrm{HfO}_{2}$ [14], etc. Graphene produced without metal catalysts is nanocrystalline, and therefore the carrier mobility is low (typically $\sim 1 \mathrm{~cm}^{2} / \mathrm{Vs}$ ) [9], and thus unsuitable for transistors. Nevertheless, they are very promising for other important applications such as transparent electrodes [16] and sensors.

In particular, the direct synthesis of graphene on standard dielectric $\mathrm{SiO}_{2}$ is one of the important goals which the semiconductor industry is pursuing [17]. This, however, has been widely found to be extremely difficult. The process is usually reported as irreproducible yielding discontinuous graphene with unknown electrical properties [17-20]. On the other hand, the formation of graphitic carbon on $\mathrm{SiO}_{2}$ has been known for several decades [21, 22], but has thus far been overlooked with regard to graphene synthesis. In this letter, we have explored the potential of this effect and demonstrated that large area uniform nanocrystalline graphene can be grown directly on oxidised Si substrates by CVD without using any metal catalysts. The growth conditions are very different from those of metal-catalysed CVD of graphene. The thickness of graphene is controllable by changing the deposition time and/or precursor partial pressure. The thin films are wrinkle-free, have no observable pinholes, and are uniform across entire wafers, as inspected by optical, scanning electron- and atomic force microscopy (SEM and AFM). Raman spectra confirm the $s p^{2}$ hybridised carbon structure. Room temperature electrical characterisation reveals ohmic behaviour and electric-field effect. Transmission electron microscopy (TEM), Raman spectroscopy and transport measurements all imply a crystalline domain size $\sim 10 \mathrm{~nm}$. The 
carrier mobility is $\sim 40 \mathrm{~cm}^{2} / \mathrm{Vs}$, which is an order of magnitude higher than previously reported for graphene grown on sapphire [9]. The transfer-free fabrication reported here demonstrates a significant step towards large scale graphene synthesis on dielectric materials and its exploitation in future applications involving transparent electrodes.

\section{Experimental}

The CVD is performed in a home-built atmospheric-pressure hot-wall quartz tube furnace. $\mathrm{CH}_{4}$ is used as a carbon precursor gas, mixed with auxiliary reduction- $\left(\mathrm{H}_{2}\right)$ and carrier $(\mathrm{Ar})$ gases. 300-nm-thick $\mathrm{SiO}_{2}$ thin films are grown by a standard wet oxidation of Si wafers (using oxyhydrogen at $1050{ }^{\circ} \mathrm{C}$ ). These substrates are heated to $1000{ }^{\circ} \mathrm{C}$ (at a rate of $\sim 30$ $\left.{ }^{\circ} \mathrm{C} / \mathrm{min}\right)$ under $\mathrm{H}_{2}(50 \mathrm{sccm})$ and $\mathrm{Ar}(1000 \mathrm{sccm})$ atmosphere and kept at $1000{ }^{\circ} \mathrm{C}$ for $3 \mathrm{~min}$. Then, $300 \mathrm{sccm} \mathrm{CH}_{4}$ is introduced to initiate the formation of graphene. Typical growth time is 30-60 min. After the deposition, the $\mathrm{CH}_{4}$ flow is stopped, leaving other gases to flow for further 3 min to remove residual reaction gases before allowing the chamber to naturally cool to room temperature $\left(\sim 20{ }^{\circ} \mathrm{C} / \mathrm{min}\right)$ in the same $\mathrm{H}_{2}+\mathrm{Ar}$ atmosphere. The nanocrystalline graphene can also be deposited directly on $\mathrm{SiO}_{2}$ by using other hydrocarbon precursors such as $\mathrm{C}_{2} \mathrm{H}_{2}$, showing the generality of the process (for details, see Supporting Information).

\section{Results and Discussion}

The graphene thin films directly grown on $\mathrm{SiO}_{2}$ are very uniform over large areas with no visible wrinkles, which can be confirmed by SEM (see 
Fig. 1). However, there are some particles on the surface, most likely due to the co-deposition of nanographite during growth. Considering that the deposition rate is low, the thickness of the graphene on $\mathrm{SiO}_{2}$ can easily be controlled while keeping high uniformity across large substrates simply by tuning the growth time. For TEM analysis, the samples are first coated with polymer (e. g. PMMA) support and subsequently immersed in diluted HF acid to separate the graphene from $\mathrm{SiO}_{2} / \mathrm{Si}$ substrates. After rinsing, the thin films are transferred to $\mathrm{Cu}$ TEM grids with a holey carbon network followed by removal of the polymer by acetone. Fig. 2 shows high-resolution TEM images of the graphene grown for $30 \mathrm{~min}$. The graphene is continuous and uniform. Nanographite is occasionally found (indicated by arrows in Fig. 2 (b)), where the layered structure of the particles is visible. In Fig. 2 (a), at the rippled/folded free-standing edge of the films, layer-by-layer structure is observed. Fig. 2 (c) shows a typical convergent beam electron diffraction pattern obtained from almost every place in the sample, which is a signature clearly indicating the hexagonal lattice structure from single-layer graphene. However, we notice that if the electron beam is moved over a distance of a few $\mathrm{nm}$ or if the beam spot is bigger than $\sim 10 \mathrm{~nm}$, diffraction patterns like Fig. 2 (d) are observed. The mixed monolayer graphene signals imply that the beam is either at domain boundaries or covers several domains. Fig. 2 provides a direct evidence of the CVD monolayer graphene and indicates a nanocrystalline grain size of $\sim 10 \mathrm{~nm}$.

Optical micrographs of the as-grown graphene are shown in Fig. 3 (a) and (b), corresponding to the growth time of 30 and 60 min, respectively. The left section of each image shows a transferred graphene grown by stan- 
dard Cu-catalysed CVD for comparison [23]. As is generally acknowledged $[6,7,16,18,19,23-25]$, and also confirmed by our Raman measurements (see Fig. 4 (a)), the graphene grown on $\mathrm{Cu}$ is primarily monolayer. In Fig. 3 (a), the two samples have almost equal colour and contrast. Thus, it is reasonable to conclude that the graphene grown on $\mathrm{SiO}_{2}$ is composed of primarily monolayer crystallites in this figure, in agreement with the TEM observation. The inset of Fig. 5 (a) shows the AFM-height profile across a step in the thin film, revealing a step height $\sim 2 \mathrm{~nm}$. Typically, a monolayer graphene fabricated by mechanical exfoliation has the AFM-measured thickness of $\sim 0.8 \mathrm{~nm}$ on $\mathrm{SiO}_{2}$, whereas after lithographic processing this thickness often increases to $\sim 1.5-2 \mathrm{~nm}[26,27]$, also in agreement with our data. However, according to Fig. 1 and Fig. 2, the coexistence of a number of few-layer graphene flakes (or nanographite) indicates that Fig. 3 is a macroscopically average effect of these flakes as well as the grain boundaries. Longer growth time leads to thicker graphene. The contrast of the samples grown during 30, 45 (not shown), and 60 min are compared with the Cu-grown graphene in Fig. 3 (c). Here, the contrast is defined as $\left(B_{s u b}-B_{g r}\right) / B_{s u b}$, where $B_{g r}$ and $B_{s u b}$ are the average brightness of the graphene and the substrate (uncovered areas in Fig. 3 (a)), respectively. Not unexpectedly, increasing the amount of $\mathrm{CH}_{4}$ in the growth chamber for a fixed deposition time also results in a thicker graphene. In the extreme case of 30 min CVD at $1000 \mathrm{sccm} \mathrm{CH}_{4}$ and $50 \mathrm{sccm} \mathrm{H}$ (as compared to our regular recipe of $300 \mathrm{sccm} \mathrm{CH}_{4}, 50 \mathrm{sccm} \mathrm{H} \mathrm{H}_{2}$ and $1000 \mathrm{sccm} \mathrm{Ar}$ in the Experimental) we obtain thick nanocrystalline graphite films (not shown). Note that the as-synthesised films retain a metallic luster even for hundreds of layers [14]. Fig. 3 is obtained by an optical microscope (Olympus) and the 
data are thus not quantitatively accurate enough to be an evidence of monolayer graphene. However, since the thickness variation in $\mathrm{SiO}_{2}$ on different chips is in the order of only $\sim 1 \mathrm{~nm}$, Fig. 3 is sufficient for a convenient estimation. We have performed variable angle spectroscopic ellipsometry (VASE) to obtain more rigorous optical information. ${ }^{0}$ The nanocrystalline graphene grown for 30 min shows qualitatively similar results as for what have been reported recently on exfoliated and Cu-grown monolayer graphene [28, 29]. Nanocrystalline graphene deposited using a similar recipe on single crystal $\mathrm{SiO}_{2}$ (quartz) also shows similar properties to standard graphene, as measured by transmission spectroscopy. ${ }^{0}$

Graphitisation is a complex physicochemical phenomenon and the detailed mechanism is not yet understood. Here, we propose two possible scenarios of the CVD of our nanocrystalline graphene. The first mechanism is a self-assembly of nanographene flakes resulting from pyrolysis of $\mathrm{CH}_{4}$ [14]. At $1000{ }^{\circ} \mathrm{C}$, most of $\mathrm{CH}_{4}$ molecules decompose. The released carbon atoms readily arrange themselves in aromatic rings and planar $s p^{2}$ hybridised graphitic layers forming nanographene crystallites up to 10 nm in size. Under usual conditions, the nanographene chaotically aggregates into bigger porous lumps with rough surfaces such as carbon black [30, 31]. In our case, a hot flat substrate forces the nanographene to orient itself parallel to the substrate thereby initiating the growth of textured thin films. The high substrate temperature and presence of $\mathrm{H}_{2}$ favour larger crystallites at the surface as they are thermodynamically more stable, while the smaller

\footnotetext{
${ }^{0}$ Sun J et al. Unpublished.
} 
ones (thin-film nuclei) are easier to decompose or react with $\mathrm{H}_{2}$. However, the small crystallites may survive at lower temperature, hereby breaking the self-assembly process that results in the ordered structure of the thin film. Indeed, at the lower-temperature zones of the CVD reactor tube we only observe porous deposits reminiscent of soot. We anticipate that this mechanism would permit growing continuous nanocrystalline graphene on virtually any dielectric substrate that withstands $\sim 1000{ }^{\circ} \mathrm{C}$ [14]. The second mechanism of the CVD of graphene on $\mathrm{SiO}_{2}$ might be of catalytic nature. The catalytic graphitisation by $\mathrm{SiO}_{2}$ powder was observed previously [21]. It is explained by the formation and decomposition of surface carbide intermediates [21] and is presumably related to the fact that $\mathrm{Si}$ can catalyse graphitisation [32]. In our experiments on bare $\mathrm{Si}$, nanocrystalline graphene can indeed be easily obtained at merely $700{ }^{\circ} \mathrm{C}$ (see Supporting Information).

The Raman spectra of graphene grown on $\mathrm{Cu}$ and nanocrystalline graphene grown on $\mathrm{SiO}_{2}$ are shown in Fig. 4. The $\mathrm{G}$ and 2D bands located at $\sim 1591$ $\mathrm{cm}^{-1}$ and $\sim 2683 \mathrm{~cm}^{-1}$, respectively, are clearly seen for all samples. These two peaks are characteristic spectral features of graphitic $s p^{2}$ hybridised materials. The well-defined peaks differentiate the as-produced nanocrystalline graphene from amorphous carbon $(a-\mathrm{C})$ [33]. Typically, the Raman spectra of $a$-C have very broad $\mathrm{G}$ and $\mathrm{D}$ bands merged together, and the $2 \mathrm{D}$ band is absent, as summarized by two groups $[34,35]$. In fact, atomically thin $a-\mathrm{C}$ films have only recently been made by bombarding graphene with electron beam [36]. In Fig. 4 (a), the 2D-to-G peak-height ratio is $\sim 2$ and the full width at half maximum (FWHM) of the symmetric 2D peak is $\sim 37 \mathrm{~cm}^{-1}$. This implies that the Cu-grown graphene is indeed a high-quality monolayer 
[7]. In comparison, the $\mathrm{SiO}_{2}$-grown graphene has higher $\mathrm{D}$ peaks at about $1350 \mathrm{~cm}^{-1}$, as seen in Fig. 4 (b). The G+D band (high-order Raman signals) at $\sim 2941 \mathrm{~cm}^{-1}[37,38]$ is also detected. Raman D band is a fingerprint of disorder in the $s p^{2}$ network of carbon materials. The $\sim 10 \mu \mathrm{m}$ laser spot in our Raman measurements covers numerous graphene domains with random in-plane orientations resulting in a strong D peak. By analysing the $I_{D} / I_{G}$ intensity ratio, disorder in the graphene monolayer can be (roughly) quantified. Using the model proposed by Lucchese et al. [33, 39], the average distance between defects is estimated to be $7-8 \mathrm{~nm}, i$. e. consistent with the graphene grain size of $\sim 10 \mathrm{~nm}$ determined by TEM and transport measurements (see below).

Hall bar structures are patterned on the as-synthesised thin films by conventional photolithography using S1813 photoresist. As electrode materials, $5 \mathrm{~nm} \mathrm{Cr}$ and $45 \mathrm{~nm} \mathrm{Au}$ are deposited by evaporation. A typical optical micrograph of the completed device is shown in the inset of Fig. 5 (b). All electrical measurements shown in this figure are performed at room temperature in air without sample annealing. Voltage $V$ is applied between the two horizontal contacts $(1,4)$ while recording the current $I$. The other electrodes permit four-terminal measurements over $4 \times 4 \mu \mathrm{m}^{2}$ of the active area of the device. The transport properties are similar for all devices on the same chip, highlighting the reproducibility of the synthesis process and the uniformity of the thin films. Fig. 5 (a) plots the $I-V$ curves of devices made from samples grown during various times. Linear (ohmic) behaviour is observed for all samples, including the contact resistances to metal electrodes. The sheet resistances $R_{s}$ obtained in the four-probe measurements 
are $13.3,6.8$, and $5.4 \mathrm{k} \Omega / \square$ for the samples grown during 30, 45, and $60 \mathrm{~min}$, respectively, $i$. e. $R_{s}$ decreases as the film thickness increases. The value for the 30-min-grown sample is larger than but comparable to that of the Cu-produced monolayer graphene [25]. Fig. 5 (b) depicts the field effect in the graphene. Back-gate voltage $V_{g}$ is applied to the conducting underlying Si substrate, which is capacitively coupled to the thin films via $300-n m-$ thick $\mathrm{SiO}_{2}$. For the 30-min-grown sample, $R_{s}$ varies by approximately $13 \%$ under $\pm 20 \mathrm{~V}$ gate voltages, while a weaker field effect is seen in samples grown for longer times. The Dirac point is not observed at this $V_{g}$ range, possibly due to the charge doping effects from the photoresist [40]. Recently, it is found that high-temperature annealing can reduce the distance between exfoliated graphene flakes and $\mathrm{SiO}_{2}$ substrates, thereby increasing the coupling between them, and leading to heavy hole doping and severe mobility degradation [27]. This can account for our reduced gating performance, since the graphene is synthesised in a long-time high-temperature process.

The Hall measurements have been carried out on the device shown in the inset of Fig. 5 (b). At both room- and low temperatures, the Hall mobility is $\sim 40 \mathrm{~cm}^{2} / \mathrm{Vs}$, an order of magnitude improvement compared with the previously reported best result for nanocrystalline graphene thin films [9]. The value is also in good agreement with the mobility extracted from the gate measurements shown in Fig. 5 (b) $[41,42]$. Based on the Hall-effect and magnetoresistance studies, independent estimation of the graphene grain size can be obtained. The results are shown in Fig. 6 where the magnetoconductance (MC) $\Delta \sigma(B)=R_{s}^{-1}(B)-R_{s}^{-1}(0)$ is presented for several temperatures from 3.8 to $290 \mathrm{~K}$. In these experiments, $R_{s}$ and the Hall resistance $R_{H}$ 
have been determined by taking the symmetric- and antisymmetric components of the voltage $V_{25}$ across contacts 2 and 5 while applying the current $I=1-10 \mu \mathrm{A}$ between contacts 1 and $4: R_{s}=\left[V_{25}(B)+V_{25}(-B)\right] / 2 I$ and $R_{H}=\left[V_{25}(B)-V_{25}(-B)\right] / 2 I$ (see Fig. 5). The MC is positive and non-zero even at room temperature. The negative magnetoresistance is characteristic for many disordered materials [43], and in particular, for carbon-based systems [44-47]. Negative magnetoresistance is usually explained by the weak localisation of carriers with some peculiarities which are characteristic for graphene $[44,48]$ (and references therein). The electron mean free path $l$ in our thin films is clearly small, because $e . g . R_{s}$ is close to the quantum resistance $R_{Q}$ at low temperature meaning that $k_{F} l \lesssim 1$, where $k_{F}$ is the Fermi wave vector. Then, the electron localisation is rather strong and the conductivity can be described by the variable-range hopping model [49] with $\ln (\sigma(T)) \backsim T^{-1 / 3}$ in the two-dimensional case. This is consistent with $R_{s}(T)$ shown in the inset of Fig. 6. It has been argued that the weak-localisation analysis can be used even in the case $k_{F} l \lesssim 1$ [45]. Indeed, our $\Delta \sigma(B)$ data can be fitted very well by the weak-localisation equation: $\Delta \sigma(B)=$ $e^{2}\left[3 F\left(4 e L_{1}^{2} B / \hbar\right)-F\left(4 e L_{0}^{2} B / \hbar\right)\right] /(2 \pi h)$, where $F(x)=\psi(0.5+1 / x)+\ln (x)$ and $\psi(x)$ is the digamma function, with two cumulative fitting parameters, $L_{0}$ and $L_{1}$, which are the characteristic singlet and triplet dephasing lengths, also including spin effects $[45,48]$ (see Fig. 6). Both $L_{0}$ and $L_{1}$ are about 10-11 $\mathrm{nm}$ at low temperature decreasing to $6-7 \mathrm{~nm}$ at room temperature. However, the accuracy of experimental $\sigma(B)$ is not sufficient to firmly extract the spin-related components of $L_{0}$ and $L_{1}$. 


\section{Summary}

We have demonstrated that atomically thin nanocrystalline carbon films can be fabricated on standard $\mathrm{SiO}_{2}$ dielectric by direct CVD without metallic catalysts. The uniform morphology of the graphene is observed by optical microscopes as well as SEM, AFM and TEM. The $s p^{2} \mathrm{C}$ network is confirmed by Raman measurements. The growth mechanisms of the thin films are briefly discussed. The as-deposited graphene shows ohmic behaviour and electric-field effect at room temperature. The disorder-induced negative magnetoresistance is observed. TEM, Raman and transport analysis all agree with the graphene nanocrystallites being in the size range of $\sim 10 \mathrm{~nm}$. We anticipate that our results will stimulate further investigation on the use of oxides in the CVD of graphene. The transfer-free process detailed here favours the industrialisation of graphene technology and hints at a promising future in a wide variety of applications such as transparent electrodes and other applications relying on cheap and chemically stable ultrathin conducting coatings.

\section{Supplementary data}

Supplementary data associated with this article can be found, in the online version, at doi:. It includes details of nanocrystalline graphene grown on $\mathrm{SiO}_{2} / \mathrm{Si}$ and $\mathrm{Si}$ from $\mathrm{C}_{2} \mathrm{H}_{2}$ precursor.

\section{Acknowlegements}

We thank J. Svensson (Lund University) and H. Jaksch (Carl Zeiss) for their generous help. Financial support from the Swedish Research Council and the Swedish Foundation for Strategic Research is appreciated. The 
clean-room processing involves the equipment sponsored by the Knut and Alice Wallenberg Foundation. J. Liu acknowledges the financial support from the Chalmers area of advances "Production". T. J. Booth and P. Bøggild acknowledge financial support from the Danish Research Council. 


\section{References}

[1] Kuzmenko AB, van Heumen E, Carbone F, van der Marel D. Universal optical conductance of graphite. Phys. Rev. Lett. 2008, 100, 117401.

[2] Schwierz F. Graphene transistors. Nature Nanotechnol. 2010, 5, 487496.

[3] Bonaccorso F, Sun Z, Hasan T, Ferrari AC. Graphene photonics and optoelectronics. Nature Photon. 2010, 4, 611-622.

[4] Novoselov KS, Geim AK, Morozov SV, Jiang D, Zhang Y, Dubunos SV, et al. Electric field effect in atomically thin carbon films. Science 2004, 306, 666-669.

[5] Miller DL, Kubista KD, Rutter GM, Ruan M, de Heer WA, First PN, et al. Observing the quantization of zero mass carriers in graphene. Science 2009, 324, 924-927.

[6] Bae S, Kim H, Lee Y, Xu X, Park J-S, Zheng Y, et al. Roll-to-roll production of 30-inch graphene films for transparent electrodes. Nature Nanotechnol. 2010, 5, 574-578.

[7] Li X, Cai W, An J, Kim S, Nah J, Yang D, et al. Large-area synthesis of high-quality and uniform graphene films on copper foils. Science 2009, $324,1312-1314$.

[8] Kim KS, Zhao Y, Jang H, Lee SY, Kim JM, Kim KS, et al. Large-scale pattern growth of graphene films for stretchable transparent electrodes. Nature 2009, 457, 706-710. 
[9] Jerng SK, Yu DS, Kim YS, Ryou J, Hong S, Kim C, et al. Nanocrystalline graphite growth on sapphire by carbon molecular beam epitaxy. J. Phys. Chem. C 2011, 115, 4491-4494.

[10] Miyasaka Y, Nakamura A, Temmyo J. Graphite thin films consisting of nanograins of multilayer graphene on sapphire substrates directly grown by alcohol chemical vapor deposition. Jpn. J. Appl. Phys. 2011, 50, $04 \mathrm{DH} 12$.

[11] Wei D, Liu Y, Zhang H, Huang L, Wu B, Chen J, et al. Scalable synthesis of few-layer graphene ribbons with controlled morphologies by a template method and their applications in nanoelectromechanical switches. J. Am. Chem. Soc. 2009, 131, 11147-11154.

[12] Ding X, Ding G, Xie X, Huang F, Jing M. Direct growth of few layer graphene on hexagonal boron nitride by chemical vapor deposition. Carbon 2011, 49, 2522-2525.

[13] Han W, Zettl A. An efficient route to graphitic carbon-layer-coated gallium nitride nanorods. Adv. Mat. 2002, 14, 1560-1562.

[14] Sun J, Lindvall N, Cole MT, Teo KBK, Yurgens A. Large-area uniform graphene-like thin films grown by chemical vapor deposition directly on silicon nitride. Appl. Phys. Lett. 2011, 98, 252107.

[15] Rummeli MH, Bachmatiuk A, Scott A, Borrnert F, Warner JH, Hoffman $\mathrm{V}$, et al. Direct low-temperature nanographene CVD synthesis over a dielectric insulator. ACS Nano 2010, 4, 4206-4210. 
[16] Park H, Rowehl JA, Kim KK, Bulovic V, Kong J. Doped graphene electrodes for organic solar cells. Nanotechnology 2010, 21, 505204.

[17] Takami T, Ogawa S, Sumi H, Kaga T, Saikubo A, Ikenaga E, et al. Catalyst-free growth of networked nanographite on $\mathrm{Si}$ and $\mathrm{SiO}_{2}$ substrates by photoemission-assisted plasma-enhanced chemical vapor deposition. e-J. Surf. Sci. Nanotechnol. 2009, 7, 882-890.

[18] Ismach A, Druzgalski C, Penwell S, Zheng M, Javey A, Bokor J, et al. Direct chemical vapor deposition of graphene on dielectric surfaces. Nano Lett. 2010, 10, 1542-1548.

[19] Sun Z, Yan Z, Yao J, Beitler E, Zhu Y, Tour JM. Growth of graphene from solid carbon sources. Nature 2010, 468, 549-552.

[20] Hofrichter J, Szafranek BN, Otto M, Echtermeyer TJ, Baus M, Majerus A, et al. Synthesis of graphene on silicon dioxide by a solid carbon source. Nano Lett. 2010, 10, 36-42.

[21] Oya A, Marsh H. Phenomena of catalytic graphitization. J. Mat. Sci. 1982, 17, 309-322.

[22] Johansson A-S, Carlsson J-O. Crystallinity, morphology and microstructure of chemical-vapor-deposited carbon films on different substrates. Thin Solid Films 1995, 261, 52-58.

[23] Sun J, Lindvall N, Cole MT, Angel KTT, Wang T, Teo KBK, et al. Low partial pressure chemical vapor deposition of graphene on copper. IEEE Trans. Nanotechnol. 2011, in press, doi: 10.1109/TNANO.2011.2160729. 
[24] Cao H, Yu Q, Jauregui LA, Tian J, Wu W, Liu Z, et al. Electronic transport in chemical vapor deposited graphene synthesized on $\mathrm{Cu}$ : Quantum Hall effect and weak localization. Appl. Phys. Lett. 2010, 96, 122106.

[25] Li X, Cai W, Jung I, An J, Yang D, Velamakanni A, et al. Synthesis, characterization, and properties of large-area graphene films. ECS Trans. 2009, 19, 41-52.

[26] Dan Y, Lu Y, Kybert NJ, Luo Z, Johnson ATC. Intrinsic response of graphene vapor sensors. Nano Lett. 2009, 9, 1472-1475.

[27] Cheng Z, Zhou Q, Wang C, Li Q, Wang C, Fang Y. Toward intrinsic graphene surfaces: a systematic study on thermal annealing and wet-chemical treatment of $\mathrm{SiO}_{2}$-supported graphene devices. Nano Lett. 2011, 11, 767-771.

[28] Kravets VG, Grigorenko AN, Nair RR, Blake P, Anissimova S, Novoselov KS, et al. Spectroscopic ellipsometry of graphene and an exciton-shifted van Hove peak in absorption. Phys. Rev. B 2010, 81, 155413.

[29] Nelson FJ, Kamineni VK, Zhang T, Comfort ES, Lee JU, Diebold AC. Optical properties of large-area polycrystalline chemical vapor deposited graphene by spectroscopic ellipsometry. Appl. Phys. Lett. 2010, 97, 253110 .

[30] Weimer AW, Dahl J, Tamburini J, Lewandowski A, Pitts R, Bingham C, et al. Thermal dissociation of methane using a solar coupled aerosol flow reactor. Proceedings of the AIChE Annual Meeting 2000, Los Angeles, CA, USA. 
[31] Biscoe J, Warren BE. An X-ray study of carbon black. J. Appl. Phys. $1942,13,364-371$.

[32] Oya A, Otani S. Catalytic graphitization of carbons by various metals. Carbon 1979, 17, 131-137.

[33] Dresselhaus MS, Jorio A, Hofmann M, Dresselhaus G, Saito R. Perspectives on carbon nanotubes and graphene Raman spectroscopy. Nano Lett. 2010, 10, 751-758.

[34] Tamor MA, Vassell WC. Raman "fingerprinting" of amorphous carbon films. J. Appl. Phys. 1994, 76, 3823-3830.

[35] Schwan J, Ulrich S, Batori V, Ehrhardt H, Silva SRP. Raman spectroscopy on amorphous carbon films. J. Appl. Phys. 1996, 80, 440-447.

[36] Kotakoski J, Krasheninnikov AV, Kaiser U, Meyer JC. From point defects in graphene to two-dimensional amorphous carbon. Phys. Rev. Lett. 2011, 106, 105505.

[37] Tan P, Tang Y, Deng YM, Li F, Wei YL, Cheng HM. Resonantly enhanced Raman scattering and high-order Raman spectra of single-walled carbon nanotubes. Appl. Phys. Lett. 1999, 75, 1524-1526.

[38] Tan P, Hu C, Dong J, Shen W, Zhang B. Polarization properties, highorder Raman spectra, and frequency asymmetry between Stokes and anti-Stokes scattering of Raman modes in a graphite whisker. Phys. Rev. B 2001, 64, 214301. 
[39] Lucchese MM, Stavale F, Ferreira EHM, Vilani C, Moutinho MVO, Capaz RB, et al. Quantifying ion-induced defects and Raman relaxation length in graphene. Carbon 2010, 48, 1592-1597.

[40] Lara-Avila S, Moth-Poulsen K, Yakimova R, Bjornholm T, Fal'ko V, Tzalenchuk A, et al. Non-volatile photochemical gating of an epitaxial graphene/polymer heterostructure. Adv. Mat. 2011, 23, 878-882.

[41] Farmer DB, Chiu H-Y, Lin Y-M, Jenkins KA, Xia F, Avouris P. Utilization of a buffered dielectric to achieve high field-effect carrier mobility in graphene transistors. Nano Lett. 2009, 9, 4474-4478.

[42] Kim S, Nah J, Jo I, Shahrjerdi D, Colombo L, Yao Z, et al. Realization of a high mobility dual-gated graphene field-effect transistor with $\mathrm{Al}_{2} \mathrm{O}_{3}$ dielectric. Appl. Phys. Lett. 2009, 94, 062107.

[43] Bergmann G. Weak localization in thin films. Phys. Rep. 1984, 107, $1-58$.

[44] Tikhonenko FV, Horsell DW, Gorbachev RV, Savchenko, AK. Weak localization in graphene flakes. Phys. Rev. Lett. 2008, 100, 056802.

[45] Wang Y, Santiago-Avilés JJ. Large negative magnetoresistance and strong localization in highly disodered electrospan pregraphitic carbon nanofiber. Appl. Phys. Lett. 2006, 89, 123119.

[46] Zhang X, Xue QZ, Zhu DD. Positive and negative linear magnetoresistance of graphite. Phys. Lett. A 2004, 320, 471-477. 
[47] Faißt A, v Löhneysen H. Electrical resistance and magnetoresistance of pyrocarbon at low temperatures in fields up to 14 Tesla. Carbon 2002, 40, 321-327.

[48] Fal'ko VI, Keshedzhi K, McCann E, Altshuler BL, Suzuura H, Ando T. Weak localization in graphene. Solid State Commun. 2007, 143, 33-38.

[49] Mott NF, Davis EA. Electronic Process in Non-Crystalline Materials. Clarendon, Oxford, 1979. 


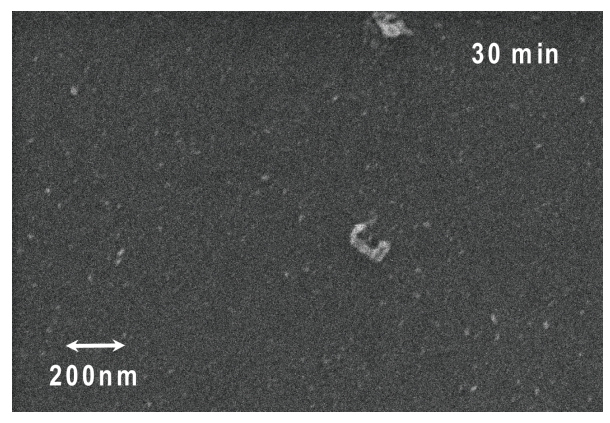

Figure 1: SEM micrograph of the nanocrystalline graphene grown for 30 min directly on $300 \mathrm{~nm} \mathrm{SiO}_{2}$. 


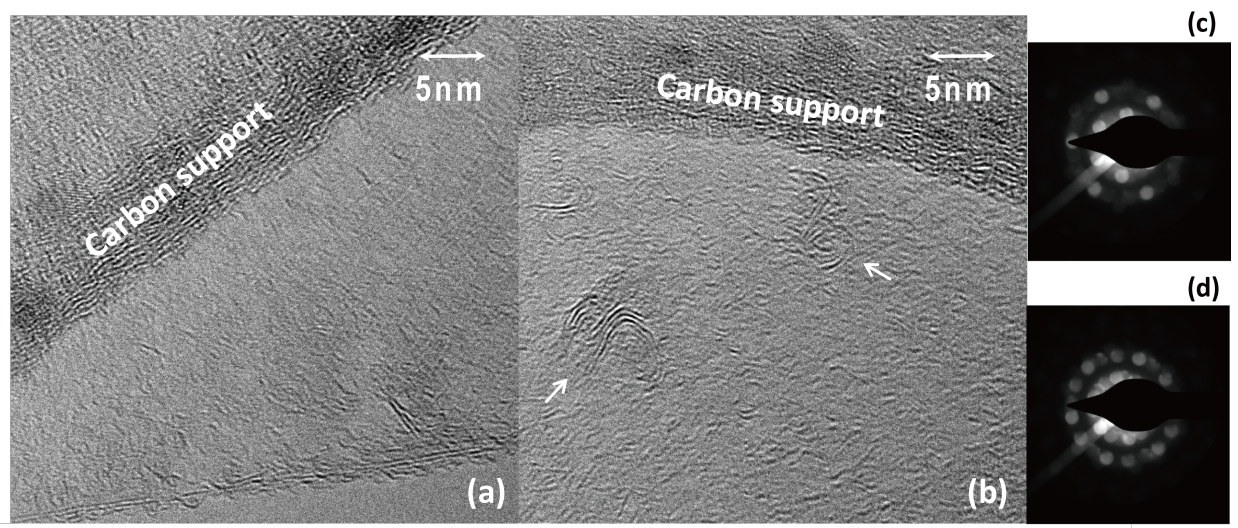

Figure 2: (a) and (b) Plan-view TEM images of the graphene directly grown on $\mathrm{SiO}_{2} / \mathrm{Si}$ for $30 \mathrm{~min}$. At the bottom of (a), a layered structure at the free-standing edge is seen, as graphene tends to roll up at free edges during transfer to TEM grids. In (b), the arrows indicate co-deposited nanographite. (c) A typical convergent beam electron diffraction pattern showing unique feature from monolayer graphene. (d) A diffraction pattern showing signals from more than one domain, in correspondence with the nanocrystalline structure. 


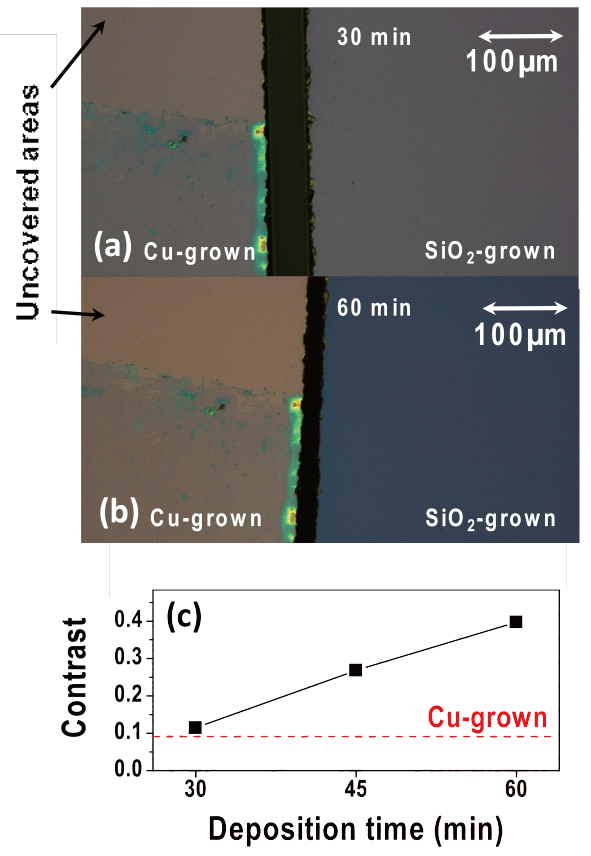

Figure 3: (a) and (b) Optical images of the graphene thin films deposited directly on $\mathrm{SiO}_{2}$ (300 nm) from $\mathrm{CH}_{4}$ precursor during 30 and $60 \mathrm{~min}$, respectively. In each micrograph, the left section is a transferred $\mathrm{Cu}$-grown graphene for comparison of optical contrast. (c) Average contrast of the graphene images versus deposition time. The dashed line indicates the contrast of the $\mathrm{Cu}$-catalysed graphene for comparison. 

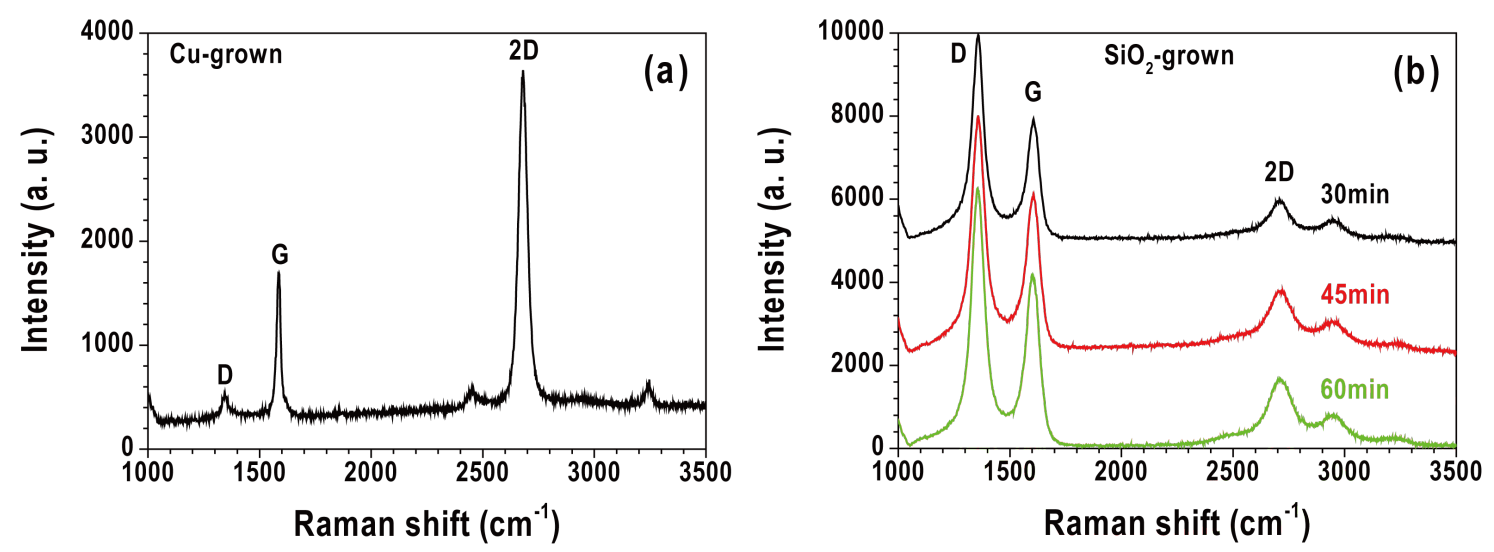

Figure 4: Raman spectra $(514 \mathrm{~nm}, \times 100$ objective, $0.5 \mathrm{~mW})$ of graphene grown by CVD. (a) Typical Raman signatures of Cu-grown graphene (transferred to $300 \mathrm{~nm} \mathrm{SiO}_{2} / \mathrm{Si}$ substrate). (b) Raman spectra of nanocrystalline graphene deposited directly on $300 \mathrm{~nm}$ $\mathrm{SiO}_{2} / \mathrm{Si}$ for 30,45 , and $60 \mathrm{~min}$. For all the samples, the $\mathrm{G}$ and $2 \mathrm{D}$ spectral peaks are clearly observed. Curves have been shifted along the ordinate for clarity. 

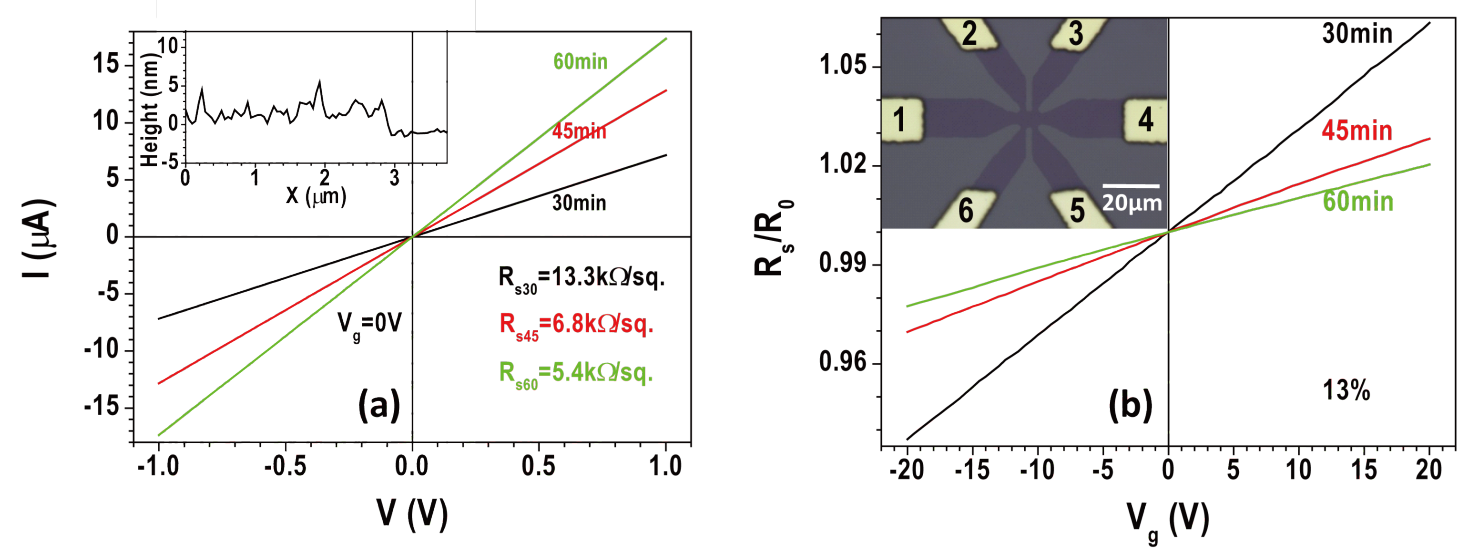

Figure 5: (a) The two-probe $I-V$ curves of devices made on samples with various deposition time. The sheet resistance $R_{s}$ is calculated from the four-probe configuration. Inset: an AFM line scan on a device made from the 30-min-grown sample, showing a step height of $\sim 2 \mathrm{~nm}$. (b) The field effect in the nanocrystalline graphene. The sheet resistances (normalised to $R_{s}$ at zero $V_{g}$ ) are plotted against the gate voltage. Inset: the optical micrograph of the device layout. The active area is $4 \mu \mathrm{m} \times 4 \mu \mathrm{m}$. 


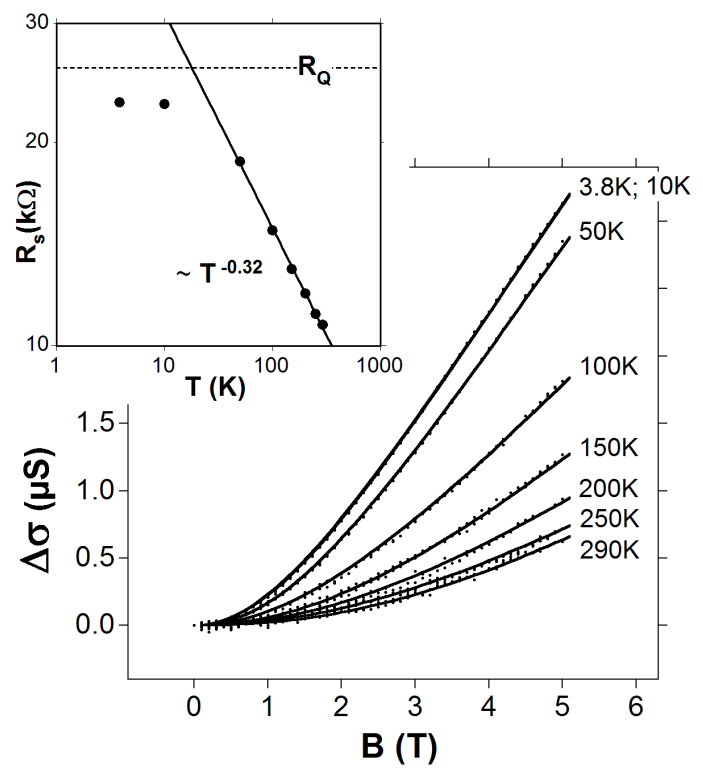

Figure 6: The magnetoconductance $\Delta \sigma(B)$ at different temperatures indicated. The inset shows the temperature dependence of the zero-field resistance $R_{s}$. The dashed line indicates the quantum resistance $R_{Q}=e^{2} / h \approx 25.8 \mathrm{k} \Omega$. The solid line is the power-low fitting for $T \geq 50 \mathrm{~K}$. 